loss function. Myotonometry is being used to quantify stiffness muscles in a valid and reliable way.

Objectives: To measure and compare the stiffness in the erector spinae muscles of axSpA patients and healthy subjects to analyse if there are any significant differences between them.

Methods: 20 axSpa patients and 20 healthy subjects matched in age, BMl and sex, were recruited. The stiffness of the lumbar erector spinae (ES) muscles was assessed at L4-5 using myotonometry (MyotonPROC) (figure 1). Measures were obtained twice in the left and right muscles, in a first test and five minutes later. $\mathrm{PRO}$ questionnaires, radiographic structural damage and metrology for axSpA were also assessed. Lumbar spinal mobility was also measured using an inertial sensor system (ViMove@).

Results: No statistical differences were found between left and right muscles neither after five minutes, so the mean values were used for analyses. All muscular measures, except Decrement showed statistically significant differences between groups. The table shows results of muscular stiffness parameters analysed, statistical differences in all parameters between groups and correlations of these parameters with conventional metrology, patient reported questionnaires (only patients) and structural damage. For control subjects, the only significant correlation was between stiffness and age. However in the axSpA patients, the myoton readings correlated with mobility measures, especially with BASMI, frontal flexion (not extension), lateral flexion and rotation, measured with the inertial sensors system. Some correlations $(p<0.05)$ were also found for BASFI and BAS-G but not for BASDAI or ASQoL.

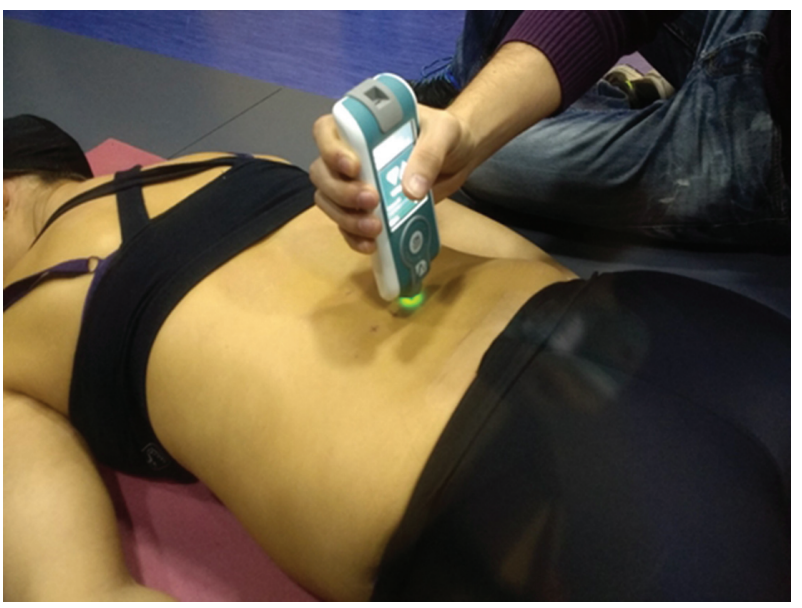

Abstract AB0868 - Figure 1

Conclusions: axSpA increases lumbar muscle stiffness with respect to healthy individuals. Muscle stiffness, as measured by myotonometry, was related to loss of movement and this could be contributing to a loss of function independently of structural damage and inflammation in axSpA. These new outcome measures could be helpful for understanding the evolution of the disease and for the functional assessment.

Acknowledgements: This study has been funded by Foreum, the XXI University of Cordoba Reearch Program and Junta de Andalucía (CS-S0029/2016).

Disclosure of Interest: None declared

DOI: 10.1136/annrheumdis-2018-eular.2238

\section{AB0869 1 PREVALENCE OF SPONDYLOARTHRITIS IN PATIENTS WITH ANTERIOR UVEITIS}

K. Bubová ${ }^{1}$, M. Gregová ${ }^{1}$, K. Zegzulková ${ }^{1}$, K. Pavelka $^{1}$, J. Heissigerová ${ }^{2}$, L. Šenolt ${ }^{1}$. ${ }^{1}$ Department of Rheumatology, Institute of Rheumatology, First Faculty of Medicine, Charles University; ${ }^{2}$ Department of Ophthalmology, First Faculty of Medicine, Charles University and General University Hospital in Prague, Prague, Czech Republic

Background: Anterior uveitis (AU) is a common extraarticular manifestation in spondyloarthritis (SpA). The disease can precede the typical axial and peripheral features. Additionally, some studies had described the imaging signs of sacroiliac involvement in patients with AU lacking chronic back pain.

Objectives: The aim of this study was to examine patients with $A U$, to determine whether the patients already fulfil criteria for axial and/or peripheral SpA and to stratify risk factors for SpA development.

Methods: We recruited 27 patients without prior rheumatologic diagnosis who developed at least one episode of AU. The clinical data were collected and rheumatology examinations were performed by trained rheumatologists. Magnetic resonance imaging (MRI) of sacroiliac joints (SIJ) was read by trained rheumatologist who was blinded to the patient data. Patients were further divided into SpA subsets (axial: imaging and clinical arm and peripheral SpA) fulfilling The Assessment of SpondyloArthritis international Society (ASAS) classification criteria $^{1}$ and non-SpA subset. The ASAS modified Berlin algorithm for diagnosis of axial $\mathrm{SpA}^{2}$ (axSpA) was also applied.

Results: Bone marrow oedema (BME) was found in $63.0 \%(n=17)$ of all patients with $\mathrm{AU}$, however $40.7 \%(\mathrm{n}=11)$ had highly suggestive $\mathrm{BME}^{3}$ corresponding to typical findings in sacroiliitis. Altogether, $22.2 \%(n=6)$ referred inflammatory back pain, $48.1 \%(n=13)$ referred non-inflammatory back pain and $29.6 \%(n=8)$ did not refer back pain. The diagnosis of SpA was confirmed in $44.4 \%(n=12)$ of al patients with $A U, 33.3 \%(n=9)$ patients fulfil the imaging arm and $7.4 \%(n=2)$ fulfi the clinical arm of ASAS classification criteria for axSpA, 7.4\% $(n=2)$ patients fulfil ASAS classification criteria for peripheral SpA (one patient fulfil both axial and peripheral criteria). The diagnosis of axSpA according to the ASAS modified berlin algorithm was confirmed in $37.0 \%(n=10)$ patients. Analysis of clinical characteristics showed significant difference between Ankylosing Spondylitis Disease Activity Score (ASDAS) in SpA vs. non-SpA (1.5 \pm 0.7 vs $0.7 \pm 0.6, p=0.01$ respectively), and remained significant in $\operatorname{axSpA}$ and also in those fulfilling only imaging arm of axial SpA (i-axSpA) $(1.4 \pm 0.7,1.3 \pm 0.7$ vs. $0.7 \pm 0.6, p=0.01$ $p=0.04$, respectively). The levels of CRP were significantly higher in SpA and axSpA compared to non-SpA subsets $(8.3 \pm 10.5,7.1 \pm 10.1$ vs. $1.8 \pm 1.6 \mathrm{mg} / \mathrm{L}$ $p=0.02, p=0.05$, respectively). Presence of back pain was increased in SpA and similarly in axSpA as well as i-axSpA compared to non-SpA subsets $(91.7 \%$ $100 \%$, as well as $100 \%$ vs. $53.3 \%, p=0.04, p=0.01, p=0.02$, respectively). Furthermore inflammatory back pain was more frequently described in axSpA and iaxSpA compared to non-SpA subsets $(45.5 \%, 44.4 \%$ vs. $6.7 \%, p=0.05, p=0.01$, respectively)

Conclusions: More than one third of patients with anterior uveitis fulfilled the criteria for axial or peripheral SpA. Furthermore, these patients had significantly higher presence of back pain, ASDAS and serum CRP levels. Inflammatory back pain was significantly increased in patients classified as axSpA.

\section{REFERENCES:}

[1] Rudwaleit, et al. Ann Rheum Dis 2009;68:777-83.

[2] Rudwaleit, et al. Ann Rheum Dis 2004;63:535-543.

[3] Rudwaleit, et al. Ann Rheum Dis 2009;68:1520-1527.

Acknowledgements: Supported by MH CR 023728, SVV 260373, AZV - 1733127A

Disclosure of Interest: None declared

DOI: 10.1136/annrheumdis-2018-eular.5295

\section{AB0870 THIRTEEN-YEAR CLINICAL FOLLOW-UP OF SPONDYLOARTRHITIS PATIENTS: DATA FROM THE REGISPONSER DATABASE}

L. Pérez Sánchez ${ }^{1,2,3}$, C. López $z^{1,2,3}$, P. Font ${ }^{1,2,3}$, L. Bautista ${ }^{1,2,3}$, M. L. Ladehesa ${ }^{1,2,3}$ I. Gómez ${ }^{1,3}$ A. Escudero ${ }^{1,2,3}$, E. Collantes ${ }^{1,2,3}$, M.C. Castro 1,2,3 ${ }^{1}$ Reina Sofía University Hospital of Córdoba; ${ }^{2} I M I B I C ;{ }^{3}$ University of Córdoba, Cordoba, Spain

Objectives: To describe the clinical characteristics of the SpA patients included in the REGISPONSER database during 13 years of follow-up, regarding the evolution of the clinical manifestations and the treatment used.

Methods: An observational and analytical study of a retrospective cohort was carried out, in which 78 patients were analysed at the Reina Sofía University Hospital of Córdoba for the first time in the year 2004/05. The last visit registered was reviewed during the years 2016/17. Data about his clinical condition, including extra-axial manifestations, and treatment was collected.

Results: Seventeen out of $78 \mathrm{SpA}$ patients included in the registry were lost. The characteristics of this cohort are shown in table 1. Recurrence of uveitis was observed in 10 out of $14(71.4 \%)$ patients $(p<0.001)$, with a low recurrence at the end of the follow-up (5\%). At the time of inclusion, uveitis was not present in 45 SpA patients, however was present in 8 of them (17.8\%) during wollow-up. Three out of the 15 patients who had history of lower limbs peripheral arthritis in the first registry, presented new flares at the end of the study, showing a low level of recurrence $(3 \%)$. During follow-up, enthesitis was present in 8 out of $45 \mathrm{SpA}$ patients $(17.8 \%)$ who didn't have a previous history of enthesitis. In adittion, only one episode of dactilitis was detected from the $55 \mathrm{SpA}$ patients who didn't have a previous history of dactilitis. The last CRP value was mean of $5.90(8.13) \mathrm{mg} / \mathrm{dl}$. Besides, three patients required placement of a hip prosthesis during follow-up. At last visit, we found a response to NSAIDs in 54 patients (88.5\%), 33 of them $(61.1 \%)$ with daily treatment. Biological therapy was present in $25 \%$ of the patients, ${ }^{15}$ showing a mean time of $18.8(7.4)$ years from the appearance of the first symptoms until their indication. A good response was achieved in $50 \%$ of 